\title{
Dificultades para la colaboración en investigación entre cardiólogos y médicos de urgencias
}

\section{Difficulties for research collaboration between cardiologists and emergency service doctors}

\author{
J. García ${ }^{1}$, F. J. Mellado ${ }^{2}$, F. Rosell ${ }^{1}$
}

\section{Sr. Director:}

Hemos leído con atención el artículo del Dr. Miró ${ }^{1}$ sobre la necesidad de colaboración entre cardiólogos y médicos de urgencia. Compartiendo, como no podía ser de otra forma, el fondo de la cuestión, nos ha parecido que no aborda algunos de los matices que influyen en esta colaboración, en concreto con los servicios prehospitalarios de emergencias (SEM).

Los SEM no son servicios finalistas. Apenas intervienen una hora escasa desde que el paciente contacta con ellos hasta se produce la trasferencia al hospital. Después, en muchas ocasiones, no vuelven a tener información sobre el resultado final. Sin embargo, esta labor intermedia es clave en el Síndrome Coronario Agudo (SCA) especialmente con elevación de ST (SCACEST). A pesar de ello, es poco frecuente encontrar en la literatura una producción firmada conjuntamente por profesionales de los SEM y cardiólogos. Lo más habitual es encontrar publicaciones sobre organización, estrategias y/o resultados firmadas por cardiólogos ${ }^{2}$ o por responsables de unidades coronarias, dependiendo de quién está a cargo del ingreso de esos pacientes ${ }^{3}$. Por otra parte, como si se tratase de procesos distintos, encontramos análisis intermedios aportados por los $\mathrm{SEM}^{4}$. Incluso cuando se hacen análisis de situación sobre la mortalidad por infarto en España ${ }^{5}$ suele olvidarse que el proceso comienza cuando el paciente contacta con el sistema sanitario. Se muestran unos porcentajes de mortalidad que no contemplan el proceso de asistencia desde su origen, lo que favorece una interpretación sesgada de la realidad asistencial y de los resultados finales en salud $^{6}$. Como bien recoge el autor, la extensión de los programas de angioplastia primaria está favoreciendo un trabajo en red $\mathrm{y}$, sin duda, unos mejores resultados. Todos estos programan pivotan en torno a los centros de coordinación de los SEM como eje central de la activación de esos códigos de actuación. La gran pega a esta colaboración es que no acaba de extenderse esa filosofía a la explotación conjunta de resultados y a la producción científica. Es además de una cuestión de justicia y de reconocimiento, un elemento clave para que los programas funcionen y mejoren a medio y largo plazo, como bien conoce el Dr. Miró .
1. Empresa Pública de Emergencias Sanitarias, Almería, España

2. Servicio de Urgencias, Hospital El Toyo, Almería. España

\section{Correspondencia:}

Fernando Rosell Ortiz

Empresa Pública de Emergencias Sanitarias

Carretera de Ronda 226, $6^{\mathrm{a}}$ planta

04007 Almería. España

E-mail: frosell@al.epes.es 
Solo desde una visión conjunta podemos contemplar el problema de salud completo y solo con un conocimiento y reconocimiento real por parte de todos los actores estos programas interniveles pueden entrar en procesos de mejora continua. Nos hubiera gustado encontrar una llamada específica a esa colaboración completa.

\section{BIBLIOGRAFÍA}

1. MIRó Ò. Necesidad de colaboración en investigación entre cardiólogos y médicos de urgencias. An Sis San Navar 2014; 37:9-16.

2. Valdés Chávarri M, Pinar Bermúdez E, Lacunza Ruz J et al. Modelo de intervención coronaria percutánea primaria en la Región de Murcia. Rev Esp Cardiol Supl 2011; 11(C): 28-34

3. Reina Toral A, Colmenero Ruiz M, García Pérez C, Expósito Ruiz M, De Antonio Martín E, Bermúdez TAmayo $C$ et al. Diferencias en los resultados de la atención a los pacientes con síndrome coronario agudo con elevación del segmento ST (SCACEST) en función del acceso inicial a hospitales con o sin sala de hemodinámica en Andalucía. Emergencias 2014; 26:101-108.
4. Houghton García RF, González Rancaño MM, Medina Camean AB, Peláez Fernández MC, Eiroa Vallés MT, Álvarez Ordiales R et al. Análisis de tiempos en los pacientes trasladados por el SAMU para intervención coronaria percutánea primaria en el primer año de instauración del Código Infarto en el Principado de Asturias. Emergencias 2014; 26: 259-266.

5. Barrabés JA, Bardají A, JimÉnez-Candil J, Del Nogal Sáez F, Bodí V, Basterra N et al. Pronóstico y manejo del síndrome coronario agudo en España en 2012: estudio DIOCLES. doi 10.1016/j.recesp.2014.03.010

6. Rosell-Ortiz F, Mellado-Vergel FJ, FernándezValle P, González-Lobato I, Martínez-Lara M, RUIZ-MONTERO MM et al. Initial complications and factors related to prehospital mortality in acute myocardial infarction with ST segment elevation. Emerg Med J 2014 doi: 10.1136/emermed-2014-203780

7. Busca P, Ortiz E, Alba L, Avilés J, Marcellán C, Miró Ò. Efecto de una estrategia de mejora de la calidad asistencial basada en la medición sistemática de indicadores en tres procesos clínicos de alta prevalencia en urgencias y comunicación de los resultados a los profesionales. Emergencias 2014; 26: 179-187. 